\title{
Clinical Outcomes with Multikinase Inhibitors after Progression on First-Line Atezolizumab plus Bevacizumab in Patients with Advanced Hepatocellular Carcinoma: A Multinational Multicenter Retrospective Study
}

\author{
Changhoon Yoo ${ }^{a} \quad J w a$ Hoon Kim ${ }^{a}$ Min-Hee Ryu ${ }^{a}$ Sook Ryun Park ${ }^{a}$ \\ Danbi Lee $^{b}$ Kang Mo Kimb Ju Hyun Shimb Young-Suk Lim ${ }^{b}$ Han Chu Lee ${ }^{b}$ \\ Joycelyn Lee ${ }^{c}$ David Tai ${ }^{c}$ Stephen Lam Chan ${ }^{d}$ Baek-Yeol Ryoo ${ }^{a}$ \\ aDepartment of Oncology, Asan Medical Center, University of Ulsan College of Medicine, Seoul, Republic of Korea; \\ ${ }^{b}$ Department of Gastroenterology, Asan Medical Center, University of Ulsan College of Medicine, Seoul, Republic \\ of Korea; 'Division of Medical Oncology, National Cancer Center, Singapore, Singapore; dState Key Laboratory of \\ Translational Oncology, Department of Clinical Oncology, Sir YK Pao Centre for Cancer, The Chinese University of \\ Hong Kong, Hong Kong, China
}

\section{Keywords}

Hepatocellular carcinoma · Multikinase inhibitor ·

Atezolizumab · Bevacizumab · Sorafenib · Lenvatinib

\begin{abstract}
Introduction: Atezolizumab-bevacizumab is the new standard of care for first-line treatment of advanced hepatocellular carcinoma (HCC). However, the optimal sequence of therapy after disease progression on atezolizumab-bevacizumab is unclear. Methods: This multinational, multicenter, and retrospective study assessed clinical outcomes of patients with advanced HCC who received subsequent systemic therapy after progression on atezolizumab-bevacizumab between July 2016 and April 2019. Results: Among 71 patients treated with atezolizumab-bevacizumab, a total of 49 patients who received subsequent systemic therapy were
\end{abstract}

Changhoon Yoo and Jwa Hoon Kim equally contributed to this work as co-first authors.

karger@karger.com www.karger.com/lic

Karger"

BOPEN ACCESS
(C) 2021 The Author(s)

Published by S. Karger AG, Basel

This article is licensed under the Creative Commons AttributionNonCommercial-NoDerivatives 4.0 International License (CC BYNC-ND) (http://www.karger.com/Services/OpenAccessLicense). Usage and distribution for commercial purposes as well as any distribution of modified material requires written permission. included in this analysis; the median age was 60 years (range, $37-80$ ) and $73.5 \%$ were male. All patients were classified as Child-Pugh A and Barcelona-Clinic Liver Cancer stage C. Multikinase inhibitors (MKIs), including sorafenib $(n=29)$, lenvatinib ( $n=19)$, and cabozantinib $(n=1)$, were used as secondline therapy for all patients. The objective response rate and disease control rate were 6.1 and $63.3 \%$, respectively, in all patients. With a median follow-up duration of 11.0 months, median progression-free survival (PFS) and overall survival (OS) were 3.4 months (95\% confidence interval [CI] 1.8-4.9) and 14.7 months $(95 \% \mathrm{Cl} 8.1-21.2)$ in all patients. Median PFS with lenvatinib was significantly longer than that with sorafenib (6.1 vs. 2.5 months; $p=0.004$ ), although there was no significant difference in median OS (16.6 vs. 11.2 months; $p=0.347$ ). Treatment-related adverse events (TRAEs) of any grade and grade 3 occurred in 42 (85.7) and 8 (16.3\%) of patients. Common TRAEs included hand-foot syndrome $(n=$ 
$26,53.1 \%)$, fatigue ( $n=14,28.6 \%)$, hypertension ( $n=14$, $28.6 \%)$, and diarrhea $(n=12,24.5 \%)$. Conclusion: Secondline treatment with MKls, mostly sorafenib and lenvatinib, showed comparable efficacy and manageable toxicities in patients with advanced HCC after disease progression on atezolizumab-bevacizumab.

(c) 2021 The Author(s)

Published by S. Karger AG, Basel

\section{Introduction}

Hepatocellular carcinoma (HCC) is the most common type of primary liver cancer; HCC comprises $75-85 \%$ of primary liver cancers and is the fourth most common cause of cancer-related deaths worldwide [1]. Asia has one of the highest incidence rates of HCC in the world. Chronic infection with hepatitis B virus and hepatitis $\mathrm{C}$ virus is the major etiologies of HCC.

Systemic therapy is the mainstay of management for patients with intermediate stage disease who are refractory or unsuitable for transarterial chemoembolization and for patients with advanced-stage HCC. For $>10$ years, sorafenib was the only agent that demonstrated a survival benefit over placebo and was the only available first-line systemic therapy [2] till lenvatinib was approved in 2018 based on the demonstration of noninferiority of overall survival (OS) compared to sorafenib [3]. These multikinase inhibitors (MKIs) are recommended as standard first-line therapy for unresectable HCC (uHCC). Other MKIs, including regorafenib and cabozantinib, and the anti-Vascular Endothelial Growth Factor Receptor (VEGFR)-2 antibody, ramucirumab, have demonstrated survival benefits for patients who failed on first-line sorafenib [4-6].

Immune checkpoint inhibitors (ICIs) have been widely investigated for use in uHCC. The anti-PD-1 antibodies, nivolumab and pembrolizumab, showed encouraging results in phase $1 / 2$ trials and were granted accelerated approved by US FDA, but failed to meet primary endpoints subsequently in phase 3 trials $[7,8]$. The combination of atezolizumab, an PD-L1 inhibitor, with the antiVEGF antibody, bevacizumab, was investigated as firstline therapy for uHCC in the phase 3 IMbrave150 trial, based on the promising results of a phase $1 \mathrm{~b}$ study $[9,10]$. In this trial, atezolizumab-bevacizumab combination therapy significantly improved median OS (not reached vs. 13.2 months; hazard ratio $0.58, p<0.001$ ), median PFS per Response Evaluation Criteria in Solid Tumors (RECIST) version 1.1 (6.8 vs. 4.3 months; HR 0.59, $p<0.001$ ), and overall response rates (ORR) per RECIST v1.1 (27 vs. $12 \%, p<0.001)$ compared to sorafenib. Furthermore, pa- tients on atezolizumab-bevacizumab also had improved quality of life and functioning, compared to patients receiving sorafenib [10]. Atezolizumab-bevacizumab is the first therapy with a significant OS benefit over sorafenib and was approved by the US FDA in May 2020 as the firstline therapy for patients with uHCC.

With the emergence of atezolizumab-bevacizumab as a first-line treatment, the treatment landscape for uHCC has changed, and one of the pressing challenges is the selection of the optimal sequence of regimens after disease progression on first-line atezolizumab-bevacizumab. Although previously approved agents are available in daily practice, it is unknown if their efficacy and safety profiles are different from that reported in the pivotal phase III trials that led to their approval. Herein, we performed a multinational, multicenter, retrospective analysis of subsequent therapy in patients with uHCC after disease progression on atezolizumab-bevacizumab.

\section{Materials and Methods}

\section{Patients}

This retrospective analysis included patients with unresectable HCC, who were diagnosed using a pathologic or noninvasive assessment, according to the American Association for the Study of Liver Diseases criteria, and treated with systemic therapy after disease progression on atezolizumab-bevacizumab. Patients were treated at the Asan Medical Center (Seoul, Korea), the Prince of Wales Hospital (Hong Kong), and the National Cancer Center (Singapore). Clinical data regarding baseline patient characteristics, treatment history, tumor response, and survival outcomes were retrospectively obtained by reviewing medical records. This study was approved by the Institutional Review Board of each participating center (Asan Medical Center [IRB No. 2020-1214], Prince of Wales Hospital [IRB No. 2019-219], and National Cancer Center [IRB No. 2018-3046]) and was performed in accordance with the ethical standards of the institutional research committee and the latest Declaration of Helsinki.

\section{Treatment}

All study patients were previously treated with a combination of atezolizumab and bevacizumab. The atezolizumab-bevacizumab combination therapy was administered either as part of the study treatment in the phase 1 GO30140 [9] and phase 3 IMBrave 150 trials [10] or given off-trial in the daily practice setting. As arm F of the phase $1 \mathrm{GO} 30140$ trial allowed crossover to atezolizumabbevacizumab combination therapy after progression on atezolizumab monotherapy, the current study also included patients who received atezolizumab monotherapy followed by the atezolizumab-bevacizumab combination therapy. As described in prior trials $[9,10]$, atezolizumab $1,200 \mathrm{mg}$ plus bevacizumab $15 \mathrm{mg} / \mathrm{kg}$ were administered intravenously every 3 weeks.

After tumor progression on atezolizumab-bevacizumab as defined by RECIST v1.1, subsequent systemic therapy was administered at the discretion of the attending physicians. The dosing schedule of each agent followed the standard dosing regimens de- 


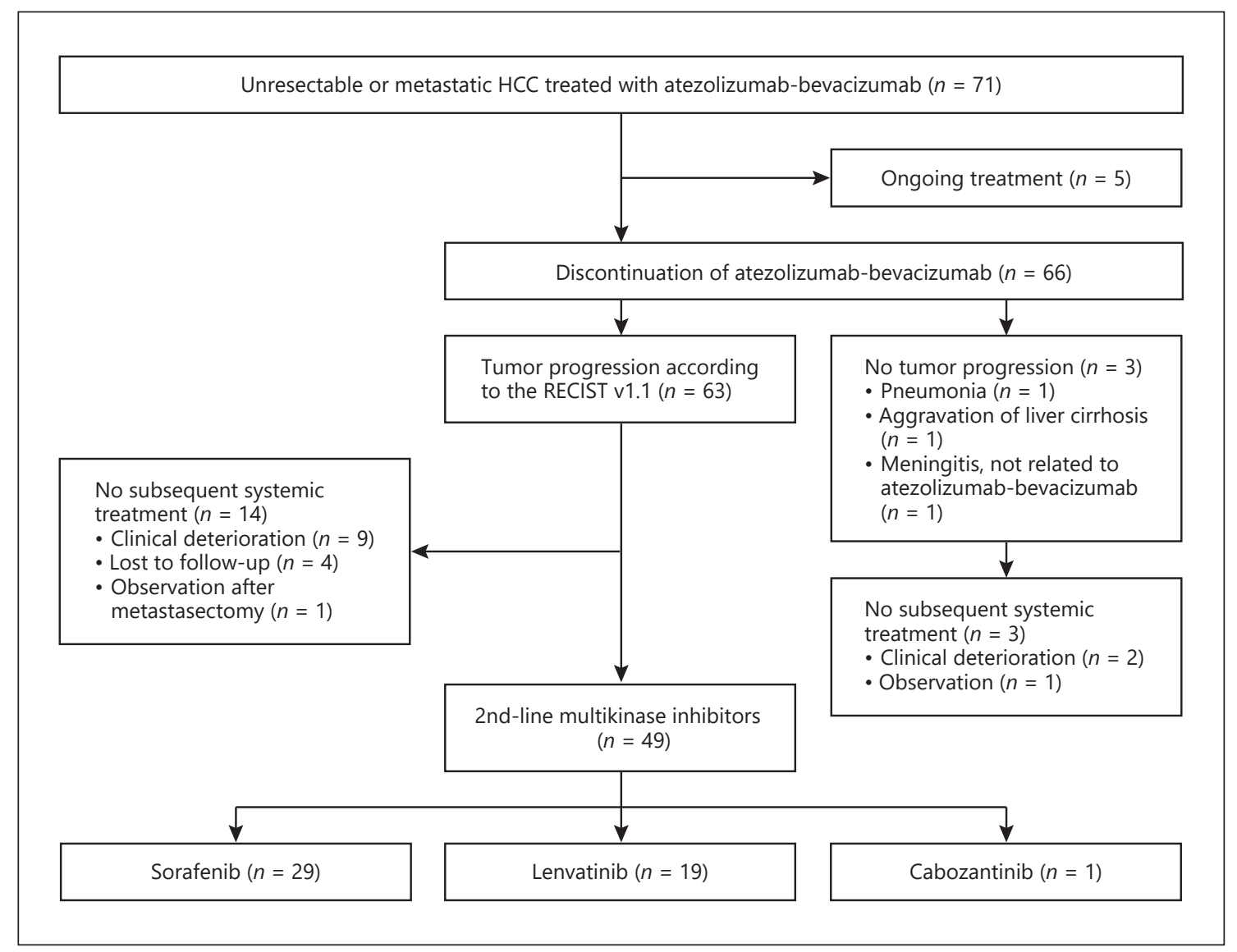

Fig. 1. Patient flow. HCC, hepatocellular carcinoma; RECIST v1.1, Response Evaluation Criteria in Solid Tumors version 1.1.

scribed in the pivotal phase 3 trials. Treatment continued until disease progression or unacceptable toxicity.

\section{Evaluation}

Tumor response was assessed every 6-8 weeks or whenever there was suspicion of disease progression using multiphase computed tomography scan and/or MRI. Tumor response was determined according to the RECIST v1.1. Adverse events were evaluated according to the National Cancer Institute Common Terminology Criteria for adverse events, version 5.0.

\section{Statistical Analysis}

Categorical and quantitative data were compared using the $\chi^{2}$ or Fisher's exact tests and Mann-Whitney U-test, respectively. The ORR and disease control rate (DCR) were assessed using RECIST v1.1. PFS was calculated from the date of initiation of second-line treatment to the date of disease progression or death from any cause, whichever came first. OS was calculated from the date of initiation of second-line treatment to the date of death from any cause. Survival curves were estimated using the Kaplan-Meier method and compared by the log-rank test. A $p$ value of $<0.05$ was considered statistically significant. The Statistical Package for the Social Sciences version 22.0 (IBM, Armonk, NY, USA) was used for all statistical analyses.

Clinical Outcomes of Subsequent Therapy

following Atezolizumab/Bevacizumab

\section{Results}

\section{Patients}

During the study period, 71 patients received atezolizumab-bevacizumab at 3 institutions and 5 patients were still under atezolizumab-bevacizumab treatment. Among them, 63 patients discontinued atezolizumab-bevacizumab due to the radiological progression according to the RECIST v1.1, while other 3 patients stopped treatment because of the reasons other than tumor progression (Fig. 1). A total of 49 patients (74.2\%) received subsequent therapy after failure of atezolizumab-bevacizum$\mathrm{ab}$ and were included in this analysis. Table 1 summarizes the baseline characteristics of the study patients. All patients received MKIs, including sorafenib $(n=29,59.2 \%)$, lenvatinib $(n=19,38.8 \%)$, or cabozantinib $(n=1,2.0 \%)$. Median patient age was 60 (range, $37-80$ ) and $73.5 \%$ of patients were male. All patients had Child-Pugh A liver function and Barcelona-Clinic Liver Cancer stage C. Most patients $(n=41,83.7 \%)$ had hepa-

Liver Cancer 2021;10:107-114 109 
Table 1. Patient baseline characteristics

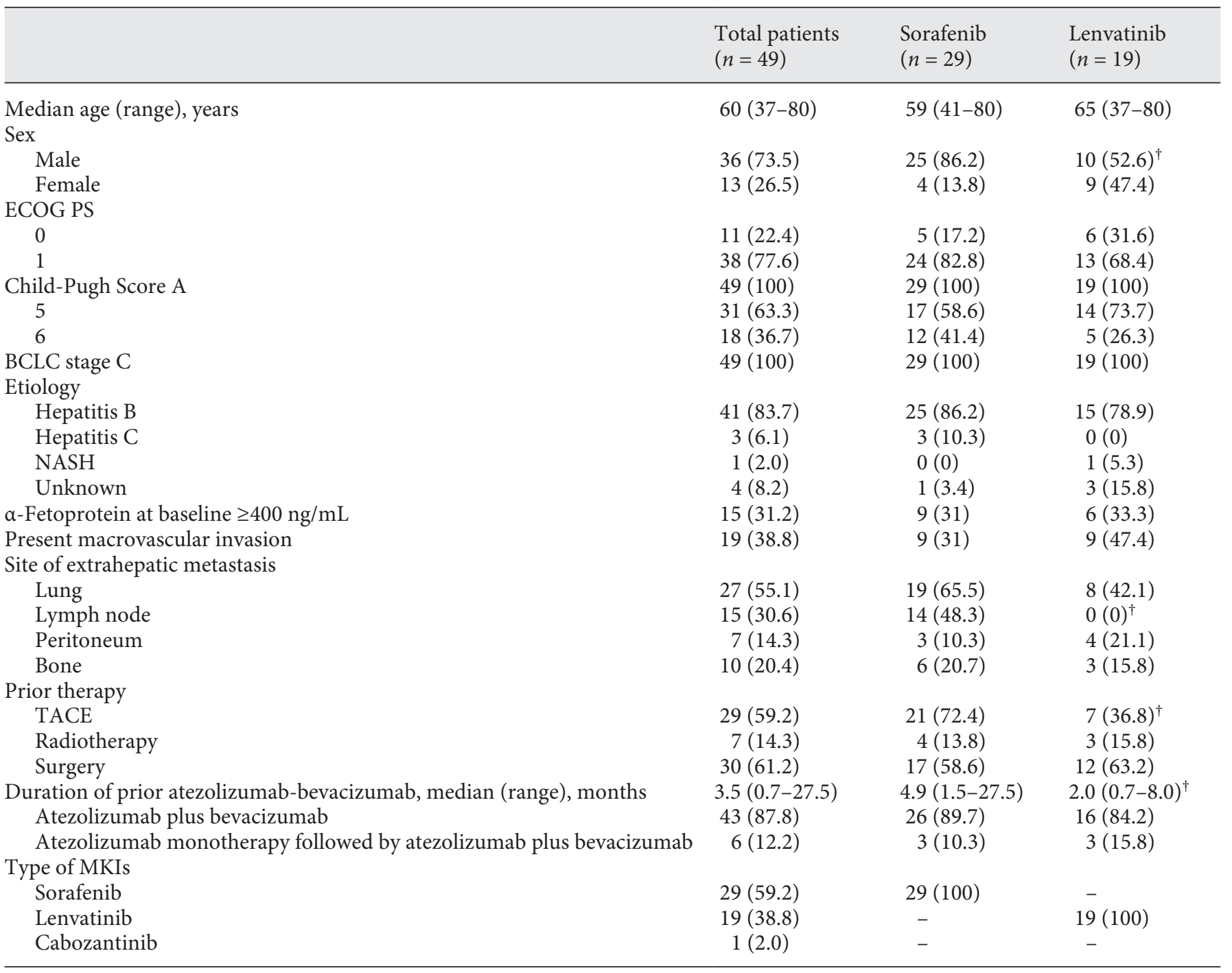

Values denote $n(\%)$ unless specified otherwise. ECOG PS, Eastern Cooperative Oncology Group performance status; BCLC, Barcelona Clinic Liver Cancer; TACE, transarterial chemoembolization; NASH, nonalcoholic steatohepatitis; MKI, multikinase inhibitor. ${ }^{\dagger}$ Significant $p$ value $(<0.05)$ using $\chi^{2}$ or Fisher exact tests and Mann-Whitney test comparing sorafenib and lenvatinib groups.

titis B virus as an etiology of HCC. The median duration of prior atezolizumab-bevacizumab was 3.5 months (range, 0.7-27.5) and 6 (12.2\%) patients received atezolizumab-bevacizumab combination following progression on atezolizumab monotherapy. The patient group receiving sorafenib had a higher proportion of males (86.2 vs. $52.6 \%, p=0.019$ ), a higher number of metastatic lymph nodes ( 48.3 vs. $0.0 \%, p<0.001$ ), an increased prior treatment history of transarterial chemoembolization (72.4 vs. $36.8 \%, p=0.019$ ) compared to the patient group receiving lenvatinib. The median duration of prior atezolizumab- bevacizumab treatment was shorter in the patient group receiving lenvatinib (2.0 [range, $0.7-8.0$ ] vs. 4.9 months [range, 1.5-27.5], $p<0.001)$ compared to the patient group receiving sorafenib. There were no other significant differences in baseline characteristics between the groups.

\section{Efficacy of Second-Line Multikinase Inhibitors}

Tumor responses to second-line MKIs are summarized in Table 2. Overall, partial response (PR), stable disease (SD), and progressive disease (PD) occurred in 3 


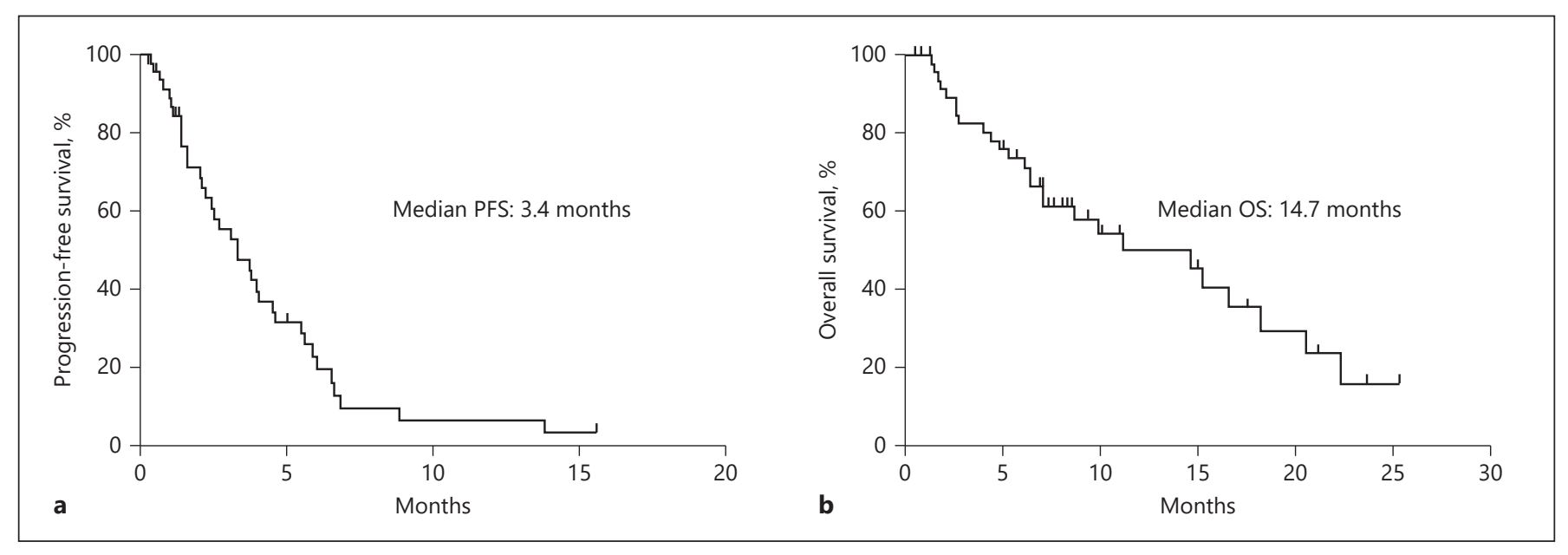

Fig. 2. The Kaplan-Meier curves of (a) PFS and (b) OS. PFS, progression-free survival; OS, overall survival.

(6.1\%), 28 (57.1\%), and $14(28.6 \%)$ patients, respectively, while there were no patients who achieved a complete response. All 3 patients who achieved PR had received lenvatinib. The ORR was $6.1 \%$ overall and tended to be higher in patients treated with lenvatinib than patients treated with sorafenib ( 15.8 vs. $0 \%, p=0.06$ ). The DCR was $63.3 \%$ overall and there was no significant difference in DCR between the 2 groups ( 62.1 vs. $63.2 \%, p=1.000)$. At a median follow-up duration of 11.0 months (95\% confidence interval [CI] 4.7-17.3), median PFS and OS were 3.4 (95\% CI 1.8-4.9) and 14.7 months (95\% CI 8.1-21.2) overall (Fig. 2). The median PFS in the lenvatinib-treated group was significantly longer than that in the sorafenib-treated group (median PFS, 6.1 months [95\% CI, 1.6-10.5] vs. 2.5 months [95\% CI, 1.3-3.8]; $p=0.004$ ). However, median OS did not differ between the lenvatinib and sorafenib groups (median OS, 16.6 months [95\% CI, 3.6-29.6] vs. 11.2 months [95\% CI, 2.7-19.6]; $p=0.347$ ) (Fig. 3).

\section{Safety Profiles}

Treatment-related adverse events (TRAEs) are shown in Table 3. TRAEs of any grade were observed in 42 (85.7\%) patients. The common TRAEs of any grade included hand-foot syndrome (HFS) $(n=26,53.1 \%)$, fatigue ( $n=14,28.6 \%)$, hypertension $(n=14,28.6 \%)$, diarrhea $(n=12,24.5 \%)$, elevated aspartate or alanine aminotransferase $(n=12,24.5 \%)$, and thrombocytopenia $(n=10$, $20.4 \%)$. Patients with sorafenib had significantly more HFS than those with lenvatinib (69.0 vs. $26.3 \%, p=0.004)$, while patients with lenvatinib seemed to have more fatigue and hypertension than those with sorafenib (fatigue; 42.1 vs. $17.2 \%, p=0.058$ and hypertension; 42.1 vs. $17.2 \%$,
Table 2. Response to MKIs according to the Response Evaluation Criteria in Solid Tumors (RECIST) version 1.1

\begin{tabular}{lcccc}
\hline & $\begin{array}{l}\text { Total } \\
(n=49)^{\mathrm{a}}\end{array}$ & $\begin{array}{l}\text { Sorafenib } \\
(n=29)\end{array}$ & $\begin{array}{l}\text { Lenvatinib } \\
(n=19)\end{array}$ & $\begin{array}{l}p \\
\text { value }\end{array}$ \\
\hline $\mathrm{PR}$ & $3(6.1)$ & $0(0)$ & $3(15.8)$ & \\
$\mathrm{SD}$ & $28(57.1)$ & $18(62.1)$ & $9(47.4)$ & \\
$\mathrm{PD}$ & $14(28.6)$ & $8(27.6)$ & $6(31.6)$ & \\
$\mathrm{NE}^{\mathrm{b}}$ & $4(8.2)$ & $3(10.3)$ & $1(5.3)$ & \\
$\mathrm{ORR}$ & $3(6.1)$ & $0(0)$ & $3(15.8)$ & 0.062 \\
DCR & $31(63.3)$ & $18(62.1)$ & $12(63.2)$ & 1.000 \\
\hline
\end{tabular}

Values denote $n(\%)$. PR, partial response; SD, stable disease; $\mathrm{PD}$, progressive disease; NE, not evaluable; ORR, objective response rate; DCR, disease control rate; MKI, multikinase inhibitor. ${ }^{a}$ One patient treated with cabozantinib achieved stable disease. ${ }^{\mathrm{b}} \mathrm{NE}$ : not evaluable $\mathrm{d} / \mathrm{t}$ loss to follow-up $(n=3)$ and too short duration of follow-up to evaluate response $(n=1)$.

$p=0.058)$. One patient treated with cabozantinib had grade 3 HFS; grade 2 diarrhea, fatigue, and thrombocytopenia; and grade 1 hypertension, anorexia, and anemia. Grade 3 TRAEs occurred in $8(16.3 \%)$ patients. The most common grade 3 TRAE was HFS $(n=4,8.2 \%)$ in both sorafenib $(n=3)$ and cabozantinib $(n=1)$ patients. There were no grade 4 TRAEs or treatment-related deaths.

\section{Subsequent Treatment}

Disease progression defined by RECIST v1.1 occurred in 36 patients (73.5\%). Among these patients, 28 (77.8\%) received subsequent systemic therapy (Table 4 ). The most commonly used agent was regorafenib $(n=16,57.1 \%)$, 


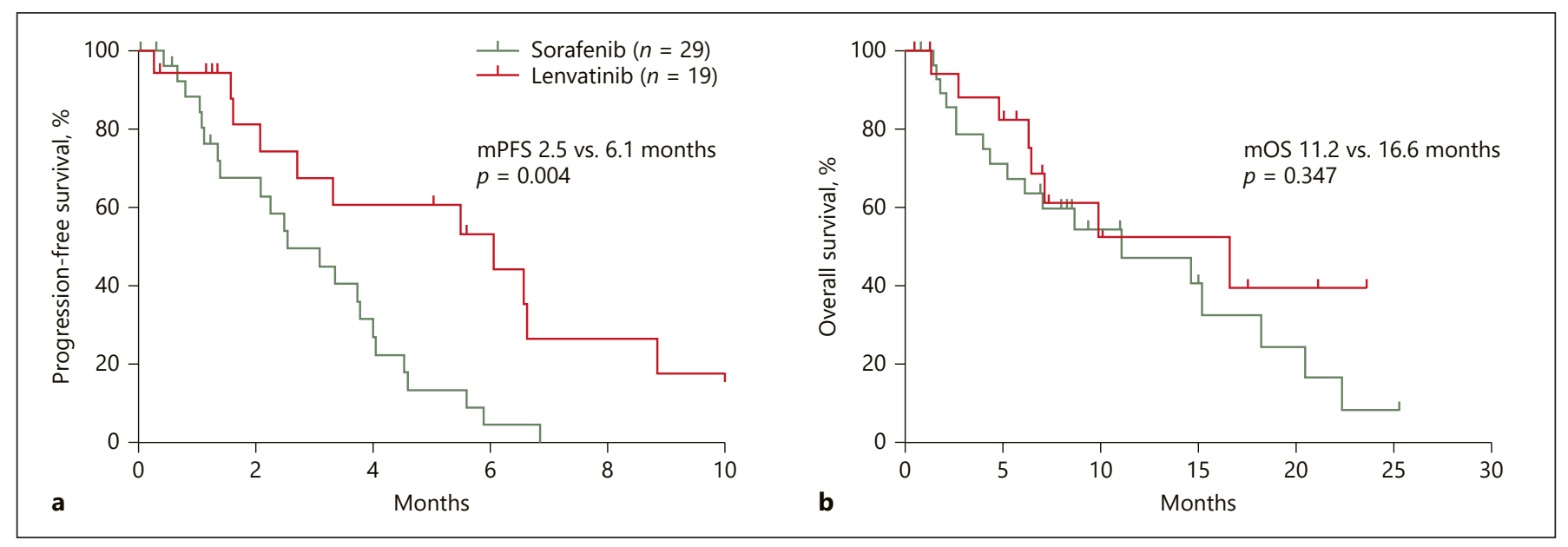

Fig. 3. The Kaplan-Meier curves of (a) PFS and (b) OS between sorafenib and lenvatinib groups. PFS, progression-free survival; OS, overall survival.

followed by sorafenib $(n=8,28.6 \%)$, nivolumab $(n=2$, $7.1 \%)$, lenvatinib $(n=1,3.6 \%)$, and ramucirumab $(n=1$, $3.6 \%)$. There was no significant difference between sorafenib and lenvatinib groups in terms of the proportion of patients who received further lines of systemic therapy ( 78.3 vs. $75.0 \%, p=0.827$ ). Most patients who progressed on sorafenib received regorafenib $(n=16$, $88.9 \%$ ), while most patients who progressed on lenvatinib received sorafenib $(n=7,77.8 \%)$. One patient treated with cabozantinib subsequently received prior sorafenib.

\section{Discussion}

In the current study, efficacy and safety of subsequent systemic therapy were evaluated in patients who progressed on first-line atezolizumab-bevacizumab. Combination therapy with atezolizumab-bevacizumab, which exhibited superior clinical outcomes compared to sorafenib, is the new standard therapy for patients with advanced HCC. All patients in this study received second-line MKI therapy, including sorafenib, lenvatinib, and cabozantinib, resulting in an ORR of $6.1 \%$ per RECIST v1.1. The median PFS was 3.4 months, and the median OS was 14.7 months. The median PFS for treatment with regorafenib and cabozantinib after failure of firstline sorafenib was 3-5 months, and the median OS was approximately 10 months $[4,5,11]$. Thus, our data indicate that subsequent MKI treatment may provide clinically meaningful efficacy in uHCC patients after progression on atezolizumab-bevacizumab.
In this study population, sorafenib (59.2\%) and lenvatinib (38.8\%), the agents previously approved as first-line therapy, were most frequently used as second-line therapy after failure of atezolizumab-bevacizumab. Efficacy outcomes of sorafenib and lenvatinib in this study were in line with the results of these agents in the prospective and real-world data as first-line therapy $[3,11,12]$. While DCR was similar between patients treated with sorafenib and lenvatinib (62.1 vs. $63.2 \%$ ), all patients who achieved PR were treated with lenvatinib and ORR per RECIST v1.1 tended to be higher with lenvatinib (15.8\%) compared to sorafenib (0\%). In addition, the median PFS was significantly better in patients treated with lenvatinib compared to those treated with sorafenib (6.1 vs. 2.5 months). However, the median OS did not differ between patients treated with lenvatinib and sorafenib. There were no differences in terms of subsequent therapy after progression on these agents. These findings (i.e., better ORR and PFS but similar OS) are similar to the results of the REFLECT trial in the first-line setting [3]. However, the results should be interpreted cautiously, as our findings are based on a small sample size with a retrospective design and there were imbalances in patient characteristics between the 2 groups.

The safety profiles of sorafenib and lenvatinib administered after first-line atezolizumab-bevacizumab were comparable to those reported in the previous phase III trials, in which these drugs were used as first-line therapies $[3,11]$. There was concern about potential unexpected toxicities, as a prior study suggested a prolonged residual immune response even after discontinuation of 
Table 3. TRASEs

\begin{tabular}{lll}
$\begin{array}{l}\text { Total } \\
(n=49)^{\mathrm{a}}\end{array}$ & $\begin{array}{l}\text { Sorafenib } \\
(n=29)\end{array}$ & $\begin{array}{l}\text { Lenvatinib } \\
(n=19)\end{array}$ \\
\hline
\end{tabular}

\begin{tabular}{|c|c|c|c|}
\hline \multicolumn{4}{|l|}{ HFS } \\
\hline Any grade & $26(53.1)$ & $20(69.0)$ & $5(26.3)^{\dagger}$ \\
\hline Grade 3 & $4(8.2)$ & $3(10.3)$ & 0 \\
\hline \multicolumn{4}{|l|}{ Diarrhea } \\
\hline Any grade & $12(24.5)$ & $6(20.7)$ & $5(26.3)$ \\
\hline Grade 3 & 0 & 0 & 0 \\
\hline \multicolumn{4}{|l|}{ Hypertension } \\
\hline Any grade & $14(28.6)$ & $5(17.2)$ & $8(42.1)$ \\
\hline Grade 3 & $2(4.1)$ & $2(6.9)$ & 0 \\
\hline \multicolumn{4}{|l|}{ Fatigue } \\
\hline Any grade & $14(28.6)$ & $5(17.2)$ & $8(42.1)$ \\
\hline Grade 3 & $1(2.0)$ & $1(3.4)$ & 0 \\
\hline \multicolumn{4}{|l|}{ Anorexia } \\
\hline Any grade & $7(14.3)$ & $2(6.9)$ & $4(21.1)$ \\
\hline Grade 3 & $1(2.0)$ & $1(3.4)$ & 0 \\
\hline \multicolumn{4}{|l|}{ Dysphonia } \\
\hline Any grade & $4(8.2)$ & $4(13.8)$ & 0 \\
\hline Grade 3 & 0 & 0 & 0 \\
\hline \multicolumn{4}{|l|}{ Skin rash } \\
\hline Any grade & $3(6.1)$ & $3(10.3)$ & 0 \\
\hline Grade 3 & 0 & 0 & 0 \\
\hline \multicolumn{4}{|l|}{ Anemia } \\
\hline Any grade & $4(8.2)$ & $1(3.4)$ & $2(10.5)$ \\
\hline Grade 3 & 0 & 0 & 0 \\
\hline \multicolumn{4}{|c|}{ Thrombocytopenia } \\
\hline Any grade & $10(20.4)$ & $6(20.7)$ & $3(15.8)$ \\
\hline Grade 3 & $1(2.0)$ & $1(3.4)$ & 0 \\
\hline \multicolumn{4}{|c|}{ Elevated aspartate or alanine aminotransferase } \\
\hline Any grade & $12(24.5)$ & $10(34.5)$ & $2(10.5)$ \\
\hline Grade 3 & 0 & 0 & 0 \\
\hline \multicolumn{4}{|c|}{ Hyperbilirubinemia } \\
\hline Any grade & $6(12.2)$ & $4(13.8)$ & $2(10.5)$ \\
\hline Grade 3 & 0 & 0 & 0 \\
\hline
\end{tabular}

Values denote $n(\%) .{ }^{\dagger}$ Significant $p$ value $(<0.05)$ using $\chi^{2}$ or Fisher exact tests comparing sorafenib and lenvatinib groups. a One patient treated with cabozantinib had grade 3 HFS; grade 2 diarrhea, fatigue, and thrombocytopenia; and grade 1 hypertension, anorexia, and anemia. TRASE, treatment-related adverse event; HFS, hand-foot syndrome.

ICIs [13]. However, there were no new safety concerns observed with the use of sorafenib or lenvatinib in the second-line setting after progression on ICIs, and the adverse events were mostly manageable with appropriate supportive care.

Effective therapeutic sequences are needed in the era of new standard first-line atezolizumab-bevacizumab therapy as the median PFS with atezolizumab-bevacizumab was 6.8 months, and the best response was PD in $19.6 \%$ of patients in the IMBrave150 trial [10]. Although
Table 4. Subsequent treatment after failure of second-line MKIs

\begin{tabular}{llll}
\hline & $\begin{array}{l}\text { Total } \\
(n=36)\end{array}$ & $\begin{array}{l}\text { Sorafenib } \\
(n=23)\end{array}$ & $\begin{array}{l}\text { Lenvatinib } \\
(n=12)\end{array}$ \\
\hline $\begin{array}{l}\text { Subsequent } \\
\text { treatment (yes) }\end{array}$ & $28(77.8)^{\mathrm{a}}$ & $18(78.3)$ & $9(75)$ \\
\hline & $(n=28,100 \%)$ & $(n=18,100 \%)$ & $(n=9,100 \%)$ \\
\hline $\begin{array}{l}\text { Regorafenib } \\
\text { Sorafenib }\end{array}$ & $16(57.1)$ & $16^{\mathrm{b}}(88.9)$ & - \\
$\begin{array}{l}\text { Nivolumab } \\
\text { Lenvatinib } \\
\text { Ramucirumab }\end{array}$ & $\begin{array}{l}2(28.6) \\
1(3.1)\end{array}$ & - & $7^{\mathrm{c}}(77.8)$ \\
\hline
\end{tabular}

Values denote $n(\%)$. ${ }^{a}$ One patient treated with cabozantinib received subsequent sorafenib. ${ }^{b}$ Among 16 patients, nivolumab $(n=6)$, lenvatinib $(n=1)$, and cabozantinib $(n=1)$ were used as further treatment. ${ }^{c}$ Among 7 patients, nivolumab $(n=2)$ was used as further treatment.

the duration of prior atezolizumab-bevacizumab was a median of 3.5 months in our study population, which was less than that shown in the IMBrave150 trial, reflecting the aggressive tumor nature, second-line MKIs showed a median PFS of 3.4 months and a median OS of 14.7 months. This relatively prolonged OS after progression on first-line therapy reinforces the importance of effective subsequent therapy. In our study, third-line systemic therapy using previously approved agents were administered in $77.8 \%$ of patients.

Currently, many combination regimens are under investigation as the first-line therapy for uHCC in randomized phase III trials [14]. In the near future, multiple combination regimens, including ICIs plus various types of targeted agents or combinations using different ICIs might become first-line therapy for the management of uHCC patients. The concept of continuum of care should be applied in the management of uHCC and planning an effective therapeutic sequence strategy from the beginning of systemic therapy is important. While there is no established biomarker for the selection of specific agents in uHCC, except ramucirumab, further real-world data and post hoc analyses may help guide physicians prior to the emergence of prospective data.

To the best of our knowledge, this is the first report investigating the subsequent therapy after progression on atezolizumab-bevacizumab in uHCC patients. However, our study has several caveats, including small sample size and a retrospective design. Multivariate analysis for a prognostic factor analysis could not be done because of 
insufficient statistical power caused by the small number of patients. Moreover, our study could not analyze the clinical outcomes of other possible second-line treatments such as regorafenib, cabozantinib, and ramucirumab, as all but 1 patient received sorafenib or lenvatinib.

\section{Conclusion}

Second-line treatment with sorafenib and lenvatinib after progression on atezolizumab-bevacizumab was as effective as the effects of these MKIs in the pivotal phase 3 trials. Further investigation to determine the optimal sequence of therapy after failure of atezolizumab-bevacizumab is warranted.

\section{Statement of Ethics}

This study was approved by the Institutional Review Board of each participating center (Asan Medical Center [IRB No. 20201214], Prince of Wales Hospital [IRB No. 2019-219], and National Cancer Center [IRB No. 2018-3046]) and has been confirmed for waiver of informed consent. This study was conducted in accordance with the Declaration of Helsinki and Good Clinical Practice.

\section{Conflict of Interest Statement}

Changhoon Yoo: Grants from Bayer and ONO; Consultancy and Advisory role for Bayer, Eisai, Ipsen, MSD, and BMS. Stephen L. Chan: Advisors for Eisai, MSD, Grants from Bayer, and MSD. David Tai: Research funding for BMS and Sirtex, honorarium from Bayer; Advisory boards for Eisai, Bayer, and Ipsen. Joycelyn Lee: Research funding from Bayer; Advisory Board for Ipsen and Bayer. The other authors have no potential conflicts of interest to declare.

\section{Funding Sources}

The authors received no specific funding for this study.

\section{Author Contributions}

Study concepts: C.Y., S.L.C., and B.Y.R. Study design: C.Y., S.L.C., and B.Y.R. Data acquisition: C.Y., M.H.R., S.R.P., D.L., K.M.K., Y.S.L., H.C.L., J.L., D.T., S.L.C., and B.Y.R. Data analysis and interpretation: C.Y., J.H.K., J.L., D.T., and S.L.C. Statistical analysis: C.Y. and J.H.K. Manuscript preparation: C.Y. and J.H.K. Manuscript editing: C.Y., J.H.K., J.L., D.T., S.L.C., and B.Y.R. Manuscript review and approval: C.Y., J.H.K., M.H.R., S.R.P., D.L., K.M.K., Y.S.L., H.C.L., J.L., D.T., S.L.C., and B.Y.R.

\section{References}

1 Yang JD, Hainaut P, Gores GJ, Amadou A, Plymoth A, Roberts LR. A global view of hepatocellular carcinoma: trends, risk, prevention and management. Nat Rev Gastroenterol Hepatol. 2019 Oct;16(10):589-604.

2 Llovet JM, Ricci S, Mazzaferro V, Hilgard P, Gane E, Blanc JF, et al. Sorafenib in advanced hepatocellular carcinoma. N Engl J Med. 2008 Jul;359(4):378-90.

3 Kudo M, Finn RS, Qin S, Han KH, Ikeda K, Piscaglia F, et al. Lenvatinib versus sorafenib in first-line treatment of patients with unresectable hepatocellular carcinoma: a randomised phase 3 non-inferiority trial. Lancet. 2018 Mar;391(10126):1163-73.

4 Bruix J, Qin S, Merle P, Granito A, Huang YH, Bodoky G, et al. Regorafenib for patients with hepatocellular carcinoma who progressed on sorafenib treatment (RESORCE): a randomised, double-blind, placebo-controlled, phase 3 trial. Lancet. 2017 Jan;389(10064): 56-66.

5 Abou-Alfa GK, Meyer T, Cheng AL, ElKhoueiry AB, Rimassa L, Ryoo BY, et al. Cabozantinib in patients with advanced and progressing hepatocellular carcinoma. $\mathrm{N}$ Engl J Med. 2018 Jul;379(1):54-63.
6 Zhu AX, Kang YK, Yen CJ, Finn RS, Galle PR, Llovet JM, et al. Ramucirumab after sorafenib in patients with advanced hepatocellular carcinoma and increased alpha-fetoprotein concentrations (REACH-2): a randomised, double-blind, placebo-controlled, phase 3 trial. Lancet Oncol. 2019 Feb;20(2):282-96.

7 Yau T, Park JW, Finn RS, Cheng A-L, Mathurin P, Edeline J, et al. CheckMate 459: a randomized, multi-center phase III study of nivolumab (NIVO) vs sorafenib (SOR) as first-line (1L) treatment in patients (pts) with advanced hepatocellular carcinoma (aHCC). Ann Oncol. 2019 Oct;30:v874-5.

8 Finn RS, Ryoo BY, Merle P, Kudo M, Bouattour M, Lim HY, et al. Pembrolizumab as second-line therapy in patients with advanced hepatocellular carcinoma in KEYNOTE-240: a randomized, double-blind, phase III trial. J Clin Oncol. 2020 Jan;38(3):193-202.

9 Lee MS, Ryoo BY, Hsu CH, Numata K, Stein $\mathrm{S}$, Verret W, et al. Atezolizumab with or without bevacizumab in unresectable hepatocellular carcinoma (GO30140): an open-label, multicentre, phase 1b study. Lancet Oncol. 2020 Jun;21(6):808-20.
10 Finn RS, Qin S, Ikeda M, Galle PR, Ducreux M, Kim TY, et al. Atezolizumab plus bevacizumab in unresectable hepatocellular carcinoma. N Engl J Med. 2020 May;382(20): 1894-905.

11 Cheng AL, Kang YK, Chen Z, Tsao CJ, Qin S, Kim JS, et al. Efficacy and safety of sorafenib in patients in the Asia-Pacific region with advanced hepatocellular carcinoma: a phase III randomised, double-blind, placebo-controlled trial. Lancet Oncol. 2009 Jan;10(1): 25-34.

12 Cheon J, Chon Hong J, Bang Y, Park Neung H, Shin Jung W, Kim Kang M, et al. Realworld efficacy and safety of lenvatinib in Korean Patients with advanced hepatocellular carcinoma: a multicenter retrospective analysis. Liver Cancer. 2020 Sep;9(5):613-24.

13 Osa A, Uenami T, Koyama S, Fujimoto K, Okuzaki D, Takimoto T, et al. Clinical implications of monitoring nivolumab immunokinetics in non-small cell lung cancer patients. JCI Insight. 2018 Oct;3(19):e59125.

14 Cheng A-L, Hsu C, Chan SL, Choo S-P, Kudo $\mathrm{M}$. Challenges of combination therapy with immune checkpoint inhibitors for hepatocellular carcinoma. J Hepatol. 2020 Feb;72(2): $307-19$. 